\title{
A Zone Assignment Algorithm for Fractional Frequency Reuse in Mobile WiMAX Networks
}

\author{
Michael Einhaus, Andreas Mäder, and Xavier Pérez-Costa \\ NEC Laboratories Europe \\ Kurfürstenanlage 36, 69121 Heidelberg, Germany \\ \{michael.einhaus, andreas.maeder, xavier.perez-costa\}@nw.neclab.eu \\ http://www.nw.neclab.eu
}

\begin{abstract}
Fractional Frequency Reuse (FFR) is a key concept for increasing the spectral efficiency of OFDMA-based broadband wireless access systems such as Mobile WiMAX. One of the main challenges when using FFR is to decide which mobile stations are assigned to which FFR time zone within a WiMAX MAC frame, so that the frame resource utilization efficiency is maximized while meeting the QoS demands of all users. In this paper we propose an efficient algorithm to address this problem with low computational complexity. The performance of the algorithm is compared with the optimum zone assignment. The evaluation has been conducted by means of a comprehensive simulation study of a typical Mobile WiMAX deployment scenario with various numbers of VoIP users.
\end{abstract}

Keywords: Broadband Wireless Access, WiMAX, OFDMA, FFR, QoS Traffic, Resource Scheduling.

\section{Introduction}

Fractional Frequency Reuse (FFR) is one of the key concepts proposed for the deployment of $3.9 \mathrm{G}$ and $4 \mathrm{G}$ cellular mobile radio systems. Next to other techniques such as power control and beamforming, FFR is an important means to mitigate interference in future radio networks [1]. The use of Orthogonal Frequency Division Multiple Access (OFDMA) at the air interface of broadband radio systems such as Mobile WiMAX [2] provides efficient means to support this concept. Furthermore, currently developed and extended standards such as 3GPP LTE/LTE-Advanced [3] and IEEE 802.16m [4] consider the FFR concept in order increase total cell data rates as required for ITU IMT-Advanced [5.

In conventional cellular deployment scenarios, orthogonal groups of resources (frequency channels) are used in neighboring cells, and these resource groups are reused in cells with a distance large enough from each other so that the interference is within tolerable limits [6]. The disadvantage of this concept is that the actual interference experienced by individual users from neighboring cells is not considered. Users that do not experience large inter-cell interference could use the whole frequency band instead of just a fraction orthogonal to neighboring cells, and thus increase the throughput. 
The basic idea behind FFR, also known as reuse partitioning [7, combines frequency reuse with full utilization of the frequency band in each cell. A mobile station (MS) that is close to the base station (BS) is allowed to utilize the whole frequency channel (reuse 1), while an MS at the cell edge uses only a fraction of the spectrum which is orthogonal to adjacent cells (reuse 3 in case of three orthogonal fractions of the spectrum ${ }^{1}$ ). Since inter-cell interference for an MS is less severe in the first case, this allows for high data rates for users close to the BS, and still sufficient data rates for users at cell edge. The throughput for each MS is thereby determined by the SINR (signal-to-noise-plus-interference ratio). The described concept can easily be extended to a sectorized cellular deployment as it is considered in this paper.

In Mobile WiMAX, the concept of FFR is supported by subdividing the frequency channel in the time domain into zones for frequency reuse 1 (Reuse 1 Zone) and zones for reuse 3 (Reuse 3 Zone). A fundamental problem in this context is how to assign service flows, corresponding to MSs, to the different zones. Service flows have to be assigned dynamically to the zones in such a way that QoS (quality of service) requirements are met while the amount of required resources should be minimized. Furthermore, the allocation algorithm has to exhibit low computational complexity in order to reduce costs.

Although the application of FFR in Mobile WiMAX has lately been addressed in several publications, proposals for practical zone assignment algorithms are scarce. The performance with different reuse patterns and subcarrier permutations is evaluated in [8] by means of simulations, showing that the coverage outage probability significantly increases with the number of users using full spectrum. However, the evaluation is based on the assumption that the zone assignment is performed according to the cell geometry and not based on SINR levels. A similar approach is followed in 9], although here an analytical model is used for the evaluation. Furthermore, results of an FFR simulation study with different Reuse Zone sizes and deployment configurations are presented in [10]. In that publication, Reuse Zone assignment is performed with a threshold-based approach: all users with downlink preamble SINR below a certain value are assigned to Reuse 3 Zone, while the others are assigned to the Reuse 1 Zone. The problem of minimizing resource utilization is for example mentioned in [11, but not explicitly evaluated.

In this paper, we extend the previously mentioned related work by proposing a new efficient algorithm for assigning QoS service flow (VoIP) with given throughput requirements to downlink FFR zones. This is done in a way so that the resource usage efficiency for these flows is maximized while using an algorithm with very low computational complexity. The purpose of maximizing the resource usage efficiency for the QoS service flows is to have as much resources as possible available for additional best effort (BE) service flows.

To the best of the authors knowledge there is no related work which addresses these objectives simultaneously. The performance of the proposed algorithm is

${ }^{1}$ Another notation is the reuse factor give by $1 / N$ ( 1 and $1 / 3$ in our case), describing the channel fraction assigned to each cell [6]. 
compared with an optimum zone assignment in terms of minimizing the amount of required resources while satisfying a given number of QoS service flows. The performance evaluation has been conducted by means of a comprehensive simulation study of a typical Mobile WiMAX deployment.

The next section provides a detailed description of how FFR is applied in Mobile WiMAX. The problem statement of FFR zone assignment and the proposed algorithm are presented in Section 3. The results of a comprehensive performance evaluation by means of a simulation study is given in Section 4 . The paper ends with some concluding remarks and an outlook.

\section{Fractional Frequency Reuse in Mobile WiMAX}

In Mobile WiMAX, the frequency channel is divided into successive MAC frames in the time domain. In TDD mode, the MAC frame is further subdivided in downlink (DL) and uplink (UL) subframes. A detailed description can be found in the underlying IEEE 802.16-2009 standard [12. In the following we focus on the downlink but a similar reasoning could be applied to the uplink direction.

FFR is realized by time zones in each WiMAX MAC frame 2. In the conventional approach, one part of the frame is reserved for reuse 1 (Reuse 1 Zone), and another part is reserved for frequency reuse 3 (Reuse 3 Zone), as shown in Fig. 1] for the downlink in a scenario with sectorized cells. The switching point between both zones can be adapted in each MAC frame. Since in Mobile WiMAX all resource is performed by the BS, it also has to decide which user data packets are assigned to which zone. All user data packets are transmitted within so-called service flows. A service flow is defined by the connection endpoints within BS and MS, and a set of QoS parameters such as throughput and delay.

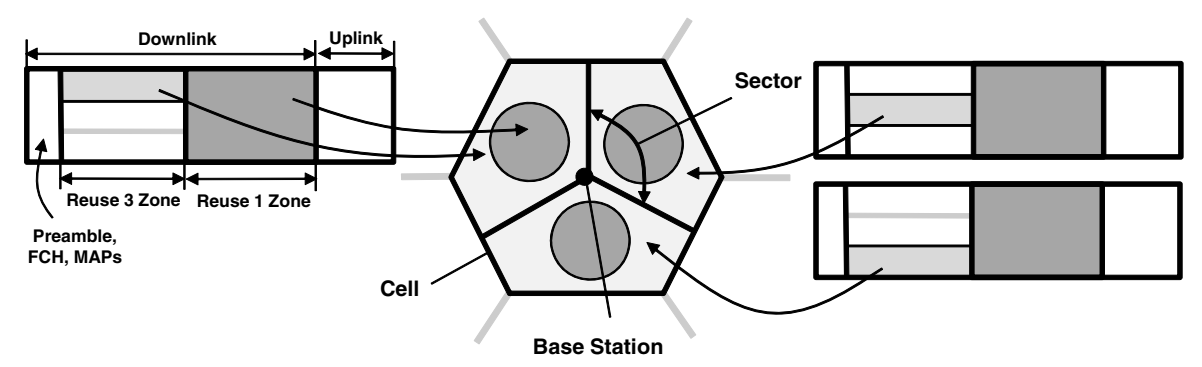

Fig. 1. FFR in a Mobile WiMAX deployment with sectorized cells

Mobile WiMAX provides different OFDMA subcarrier permutations for the formation of subchannels. For the use of FFR, PUSC (partial use of subcarriers) should be used since it provides orthogonal subchannel sets which coincide in different cells which is an inevitable prerequisite for the application of FFR. PUSC subchannels are formed by pseudo randomly distributed subcarrier sets in the frequency domain. As illustrated in 8, the same subcarrier permutation base 
has to be used by each BS in the whole network in order to provide orthogonal subchannels.

Resources are assigned to service flows in form of slots. A DL PUSC slot has the dimensions of one subchannels and two symbols. The slot capacity in terms of bits/slot is determined by the modulation and coding scheme, which is dynamically selected based on the SINR level by means of adaptive modulation and coding (AMC). A more detailed description of the system profile used in this paper is given in the performance evaluation (Section 4.2).

\section{Zone Assignment for Fractional Frequency Reuse}

\subsection{Problem Statement}

The problem that has to be solved is the assignment of a number of QoS service flows with given throughput requirements to the different Reuse Zone of the DL $\mathrm{MAC}$ frame in a way so that the amount of required resources for that service flows is minimized.

We define the number of available DL PUSC slots in Reuse 1 and Reuse 3 Zone as $S_{1}$ and $S_{3}$, respectively. Each service flow is assigned to and scheduled in one of these two zones of the DL MAC frame, depending on the corresponding MS position and channel conditions. Each service flow $k$ requires the transmission of a certain number of bits $T_{k}$ per MAC frame, which corresponds to the assignment of $R_{z, k}$ number of slots in zone $z$. Due to the use of AMC in Mobile WiMAX, this number of slots is determined by the slot capacity $C_{z, k}$ depending on the SINR level of the MS in each zone.

Based on knowledge of the SINR level of each MS (and corresponding service flows) in both zones, the BS has to decide which service flow is served in which Reuse Zone. Mathematically, the problem of zone assignment with minimum resource usage can be formulated as the following Integer program:

$$
\begin{array}{ll}
\min . & \sum_{k=0}^{N-1} R_{1, k}+\sum_{k=0}^{N-1} R_{3, k} \\
\text { s.t. } & R_{1, k} \cdot C_{1, k}+R_{3, k} \cdot C_{3, k} \geq T_{k} \quad \forall k \\
& \sum_{k=0}^{N-1} R_{z, k} \leq S_{z} \quad, \quad z \in\{1,3\} \\
& R_{1, k} \cdot R_{3, k}=0 \quad \forall k
\end{array}
$$

The given conditions state that the QoS requirements of all service flows have to be satisfied in terms of bits $T_{k}$ per MAC frame, that the sum of all assigned slots $R_{z, k}$ in each zone shall not exceed the corresponding number of available slots $S_{z}$, and that each service flows is just assigned to one zone. Since resources cannot be shared by different service flows, the number of assigned slots $R_{z, k}$ is either a positive Integer value or zero. 
The complexity of the assignment problem necessitates the development of efficient heuristics with low computational complexity, which in the best case can be customized to meet operator requirements. Furthermore, the algorithm should preferably be easy to implement.

\subsection{Proposed Algorithm}

To find the solution for the problem described in Eqs. (1), a complete search comparing all possible slot assignment patterns with complexity $O\left(2^{N}\right)$ has to be performed. To minimize the computational complexity, we propose a zone assignment algorithm that is based on the sequential assignment of service flows to preferred zone after first sorting all flows by a certain metric. Thus the complexity is determined by the sorting of $N$ service flows, which is $O(N \log N)$.

The key idea behind the proposed sorting metric is that it should not just consider zone preference from a single service flow's point of view (based on SINR levels), it should rather consider how the assignment of each flow to a certain zone would affect the total resource utilization.

In this context, the relative resource usage efficiency within a zone should be considered because the preference for a certain zone should be high for a service flow with high efficiency in that zone compared to other flows. The reason for this is demonstrated by the following simple example. Consider a service flow which has in Reuse 1 Zone an SINR level which is much larger than the average SINR level of all flows in that zone, but in the Reuse 3 Zone its SINR level is only slightly above the corresponding average. Hence, the overall system performance will benefit more from assigning that flow to the Reuse 1 Zone instead of the Reuse 3 Zone. The important feature here is that this applies even if the absolute SINR level of that flow is higher in the Reuse 3 Zone than in the Reuse 1 Zone, which is an essential difference compared to algorithms where the zone assignment is based just on the absolute SINR level.

In addition to that, the relative zone size should also be considered. In general, the average number of service flows assigned to a certain zone increases with the zone size, independently of the applied allocation algorithm. That means that the efficient utilization of zone resources in a large zone can amount to a large number of resources that are conserved compared to an inefficient allocation. The amount of resources that can be saved by efficient allocation in a small zone is compared to that normally rather small, in the worst case not even enough to bear an additional service flow.

The proposed sorting metric $\Phi_{k}$ of service flow $k$ which considers both factors (relative SINR level and relative zone size) in a multiplicative manner is determined by the following equations:

$$
\begin{gathered}
\Phi_{z, k}=\frac{\gamma_{z, k} \cdot N}{\sum_{k=0}^{N-1} \gamma_{z, k}} \times \frac{S_{z}}{S_{1}+S_{3}} \quad, \quad z \in\{1,3\} \\
\Phi_{k}=\max \left(\Phi_{1, k}, \Phi_{3, k} \cdot \alpha\right) \quad, \quad \alpha \geq 0
\end{gathered}
$$


In these equations, $N$ is the number of service flows, $\gamma_{z, k}$ is the absolute SINR level of service flow $k$ in FFR zone $z$, and $S_{z}$ is the number of available resources in the Reuse Zones ( $z$ can be 1 or 3 ), corresponding to Eqs. 11 The algorithm comprises one degree of freedom, represented by the tuning factor $\alpha$, for adjustment to a certain environment and user distribution.

In the sorted list, each service flow has a preferred FFR zone: if $\Phi_{k}=\Phi_{1, k}$, the preferred zone is the Reuse 1 Zone, otherwise it is the Reuse 3 Zone.

The tuning factor $\alpha$ is used to adapt the algorithm to the number of service flows. It will be shown in the performance evaluation why this additional factor has a significant impact on the performance. The impact of extreme settings of $\alpha$ is given by:

$$
\begin{aligned}
& \left.\Phi_{k}\right|_{S_{1}>0, \alpha=0}=\Phi_{1_{k}} \\
& \left.\Phi_{k}\right|_{S_{3}>0, \alpha \rightarrow \infty}=\Phi_{3_{k}} \cdot \alpha
\end{aligned}
$$

In the first case $(\alpha=0)$, all service flows are sorted according to the quality (SINR level) in the Reuse 1 Zone, and this zone is preferred by all flows. In the second case $(\alpha \rightarrow \infty)$, all flows prefer the Reuse 3 Zone and are sorted by the quality in that zone.

After sorting all flows in descending order of $\Phi_{k}$, the actual zone assignment is then done in the following way: beginning with the first flow in the sorted list, each flow is assigned to the preferred zone if there are enough resources available in that zone, otherwise it has to be assigned to the other zone. If not all service flows can be served, this is considered as an outage in the following.

\section{Performance Evaluation}

\subsection{Methodology}

The efficiency of the algorithm is evaluated by the total resource utilization $U_{T}(x)$ of the QoS service flows in the DL MAC frame, where $x$ is the relative zone switching point position within the frame, ranging from 0 to 1 :

$$
U_{T}(x)=\frac{\sum_{k=0}^{N-1} R_{1, k}(x)+\sum_{k=0}^{N-1} R_{3, k}(x)}{S_{1}(x)+S_{3}(x)}
$$

Corresponding to the description in Eq. 1, $R_{z, k}$ represents the amount of resources assigned to service flow $k$ in Reuse Zones $z$, and $S_{z}$ is available number of resources in the zones. With $x=0$, the whole DL MAC frame is used as Reuse 1 Zone, and with $x=1$ it is used as Reuse 3 Zone.

Furthermore, the outage probability $P_{o}(x)$ is evaluated. We define it here as the probability that at least one of the given number of the QoS service flows cannot be provided with the required data rate. This means that the amount of available resources in the DL MAC frame is not sufficient.

For the comparison of the proposed FFR zone assignment algorithm with the optimum assignment, the difference between both curves describing the expectation of $U_{T}(x)$ is evaluated by the mean squared error (MSE):

$$
E(\alpha)=\frac{1}{|\mathbf{X}|} \sum_{x \in \mathbf{X}}(O(x)-A(\alpha, x))^{2}
$$


In this equation, $O(x)$ and $A(\alpha, x)$ are the curves for the optimum given by the solution of Eqs. (11) and the proposed algorithm, respectively. $\mathbf{X}$ is the set of all evaluated zone switching points.

\subsection{Simulation Scenario}

The simulated scenario consists of 19 hexagonal cells in a wrap-around scenario with three sectors per cell, as described in the evaluation methodology for IMT-Advanced [5]. The SINR is calculated based on pathloss and shadowing. The impact of frequency-selective small scale fading is not considered in the simulation study since the performance is evaluated with distributed subcarrier permutations (PUSC in WiMAX) which in combination with channel coding minimizes the impact of frequency-selective fading due to averaging effects.

We use the ITU Suburban Macro model for pathloss and shadowing, as defined in [5]. The inter-site distance in this model is $1299 \mathrm{~m}$, and the carrier frequency is $2.0 \mathrm{GHz}$. We assume a fixed downlink transmission power of $43 \mathrm{dBm}$ and a thermal noise density of $-174 \mathrm{dBm} / \mathrm{Hz}$ for the $10 \mathrm{MHz}$ channel.

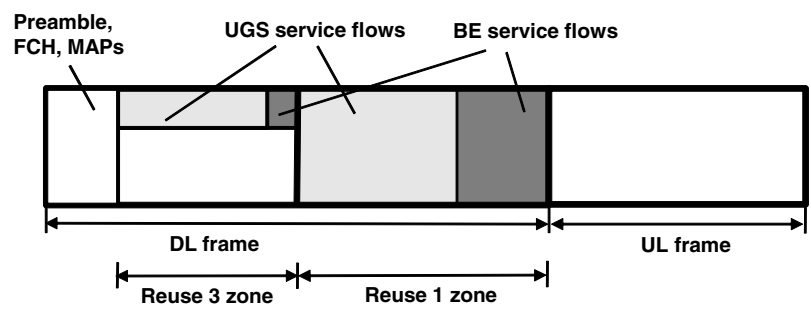

Fig. 2. Mobile WiMAX MAC frame model with DL FFR

The MAC frame has a duration of $5 \mathrm{~ms}$, and we consider a bandwidth of $10 \mathrm{MHz}$ and an FFT size of 1024 which results in 47 symbols within a frame. It is assumed that 35 OFDM symbols are reserved for downlink and 12 symbols for uplink transmissions. The first downlink symbol is used for the preamble, and four symbols are reserved for signalling in form of WiMAX FCH and MAPs [2]. The remaining 30 symbols are partitioned into DL Reuse Zones 1 and 3 . In the frequency domain the subcarriers are grouped to $30 \mathrm{DL}$ subchannels (and $35 \mathrm{UL}$ subchannels). For the application of FFR in downlink direction this means that in the Reuse 1 Zone all 30 subchannels are used, and in the Reuse 3 Zone just 10 subchannels.

As resource (slot) allocation strategy we assume that the DL MAC frame is filled up column-wise starting from the upper left corner. This corresponds to the allocation strategy which is mandatory for use of HARQ 12 and enables an one-dimensional representation of the available resource per frame.

For the QoS service flows, we assume CBR data streams (UGS service flows) of $80 \mathrm{kbit} / \mathrm{s}$. This corresponds to $64 \mathrm{kibt} / \mathrm{s}$ VoIP traffic (G.711) with a packet generation interval of $20 \mathrm{~ms}$ plus $16 \mathrm{kbit} / \mathrm{s}$ overhead due to IP, UPD and RTP 
packet headers (40 bytes per data packet each $20 \mathrm{~ms}$ ). The resources of the DL MAC frame that are not used for the transmission of CBR traffic are assumed to be completely occupied by BE traffic as shown in Fig. 2. Due to this, all resources are always used for DL data transmissions, which means that the SINR levels of the reuse zones do not depend on the allocation decision.

The mapping of SINR level to MCS given in Tab. 1 is based on the performance evaluation results presented in [13. Due to the frame duration of $5 \mathrm{~ms}$, the $80 \mathrm{kbit} / \mathrm{s}$ per VoIP service flow results in a required average transmission of 200 bits per frame.

Table 1. SINR to MCS mapping

\begin{tabular}{cccc}
\hline \multicolumn{2}{c}{ MCS } & min SINR & bits/slot \\
\hline QPSK & $1 / 2$ & $3.5 \mathrm{~dB}$ & 48 \\
16QAM & $1 / 2$ & $10.0 \mathrm{~dB}$ & 96 \\
64QAM & $1 / 2$ & $15.5 \mathrm{~dB}$ & 144 \\
64QAM & $2 / 3$ & $21.0 \mathrm{~dB}$ & 192 \\
64QAM & $3 / 4$ & $24.5 \mathrm{~dB}$ & 216 \\
\hline
\end{tabular}

For the performance evaluation, 10000 random user (VoIP service flow) placements are generated for each zone switching point setting. In a each user placement, there is a fixed uniformly distributed number of users generated per sector. All presented results show the average performance of all user placements.

\subsection{Simulation Results}

Optimum FFR Zone Assignment. Fig. 3 shows the expectation of the resource utilization $U_{T}(x)$ and the outage probability $P_{o}(x)$ depending on the zone switching point position for different numbers of uniformly distributed VoIP service flows per sector. The results reveal that there exists an optimum Reuse Zone switching point concerning the minimum average resource utilization. The larger the number of VoIP service flows, the larger is the optimum size of the Reuse 3 Zone in the DL MAC frame, represented by relative zone size $x$.

The results also show that the optimum switching point for the minimum resource utilization does not automatically minimize the outage probability. If beginning from the optimum zone switching point the Reuse 3 Zone size is further increased, this results in a further reduced outage probability at the cost of reduced efficiency in resource utilization for the VoIP service flows.

The very high outage probabilities and resource utilization values with a very small Reuse 3 Zone $(x \rightarrow 0)$ is based in the fact that in general the use of frequency reuse 1 cannot be applied due to very low SINR values at the cell edge. The resource utilization for $x=0$ corresponds to a conventional cellular deployment with frequency reuse 3 . Therefore the results clearly prove the advantage of FFR since the resource utilization can be significantly reduced, i.e. with the optimum frame partition $x_{\text {opt }}$ more than $20 \%$ in case of 16 VoIP flows. It can 


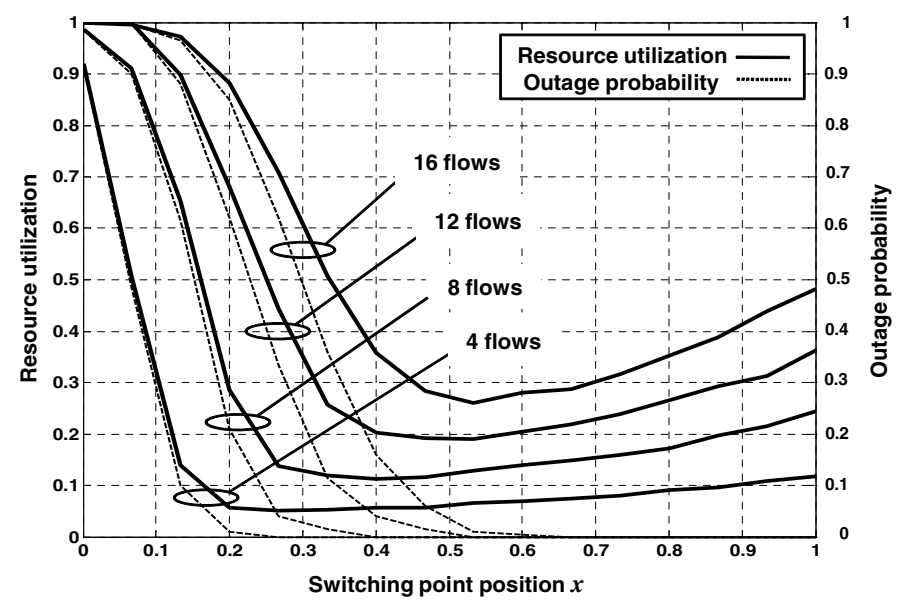

Fig. 3. Resource utilization and outage probability

be seen that FFR utilization gain increases with the number of flows which is based on exploitation of multiuser diversity.

Proposed FFR Zone Assignment Algorithm. As explained in Section 3 the proposed algorithm features a degree of freedom represented by the tuning factor $\alpha$. It should be set in a way so that the algorithm provides close to optimum performance, meaning that the mean squared error $E(\alpha)$ in Eq. (6) has to be minimized.

The impact of $\alpha$ on the performance for 8 and 14 VoIP service flows is exemplary shown in Fig. 4. The results reveal that the performance of the proposed algorithm converges to the optimum with an appropriate setting of $\alpha$. This optimum $\alpha$ depends on the number of flows; when the number of flows is increased, the magnitude of $\alpha$ has to be reduced. With 8 flows, $\alpha=8.0$ shows very good performance; and with 14 flows, the setting of $\alpha=4.0$ is more appropriate.

The results show that the proposed algorithm meets the optimum performance quite well if one FFR zone is significantly larger than the other. According to the sorting metric of the sequential assignment given by Eqs. (2) and (3), the larger FFR zone is filled first with the best service flows first in these cases. Actually, if the whole DL MAC frame is used as Reuse 1 Zone or as Reuse 3 Zone $(x=0.0$ and $x=1.0$, respectively), the performance curves coincide since anyway all flows have to be assigned to a single zone. The largest differences between optimum and $\alpha$-dependent algorithm appears in the range of medium FFR zone sizes with $0.2 \leq x \leq 0.8$. In general the differences increase with the number of flows, implying more possible assignment combinations from which the best one can be selected with the complete search approach. However, the optimum switching point in terms of minimum resource utilization almost coincides for optimum zone assignment and proposed algorithm with optimum $\alpha$ selection. 

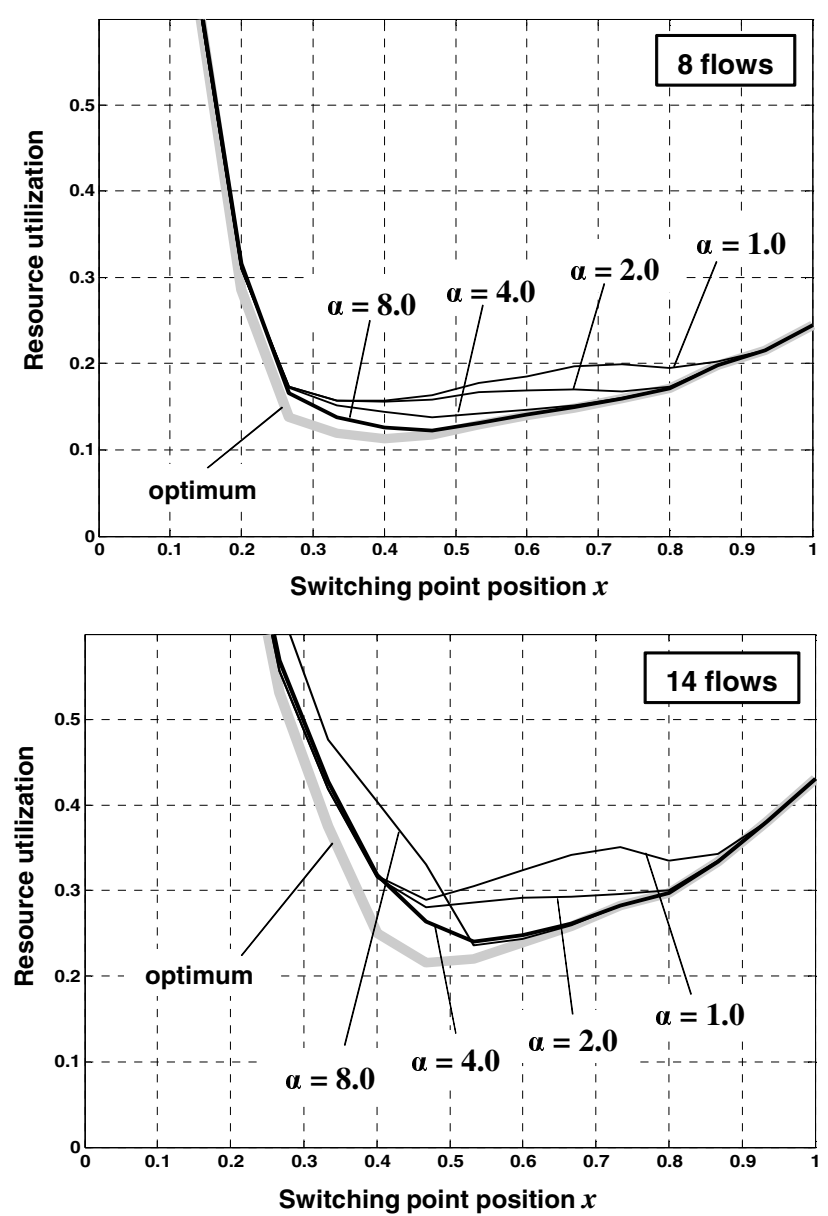

Fig. 4. Resource utilization of proposed algorithm with 8 and 14 VoIP flows

Concerning the tuning factor $\alpha$, another effect is revealed in the evaluation results (especially in case of 14 flows). If $\alpha$ has a large value, the performance of the proposed algorithm meets the optimum very good for large $x$ values; and if $\alpha$ is small, the performance is very good for small $x$ values, correspondingly.

For an in-depth analysis of the impact of $\alpha$, the difference between the optimum and the performance curve of the proposed algorithm is quantified in Fig. 5. It shows the mean squared error $E(\alpha)$, as defined in Eq. (6), for different numbers of service flows. The results exhibit that the error is determined by a convex function of tuning factor $\alpha$, which forms a fundamental finding.

Concerning the dependency on the number of service flows, the results show that the dependency of the performance on $\alpha$ increases with the number of flows. Another important finding is that the minimum achievable error $E\left(\alpha_{o p t}\right)$ increases with the number of flows. Furthermore it can be seen that the $\alpha$ 


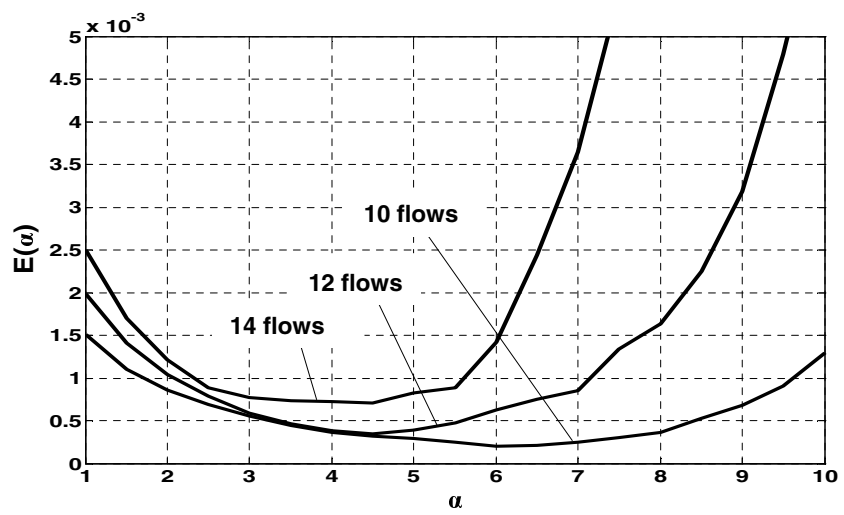

Fig. 5. Comparison of proposed algorithm with optimum

Table 2. Optimum $\alpha$ values depending on number of VoIP service flows

\begin{tabular}{l|cccccc}
\hline flows & 4 & 6 & 8 & 10 & 12 & 14 \\
$\alpha_{\text {opt }}$ & 10.0 & 9.0 & 8.0 & 6.0 & 4.5 & 4.5 \\
\hline
\end{tabular}

value providing this minimum error decreases when the number of flows is increased.

The value of this $\alpha_{o p t}$ depending on the number of VoIP service flows is given in Tab. 2. For the evaluation, the tuning factor $\alpha$ has been varied in steps of 0.5. The results show that the optimum value decreases in an approximately linear manner with the increasing number of service flows until reach saturation at 4.5. The major findings in this context are that, corresponding to Eq. (3) in the algorithms description, the mean preference for the Reuse 3 Zone should be always higher than the preference for the Reuse 1 Zone $(\alpha>1.0)$. Furthermore, the degree of the average preference for the Reuse 3 Zone in the algorithm should be reduced when the number of VoIP service flows is increased.

\section{Conclusions and Outlook}

In this paper we presented an efficient algorithm for Mobile WiMAX to assign service flows to FFR zones considering QoS needs in terms of throughput. The proposed assignment scheme provides low complexity, yet achieves close to optimum performance in terms of resource utilization efficiency. This has been proven by means of a detailed simulation study of a typical Mobile WiMAX deployment with various numbers of VoIP service flows.

The main conclusions drawn from our results are: i) an FFR algorithm exhibiting close to optimum performance in terms of resource utilization is feasible at low complexity costs, ii) the performance difference compared to the optimum is a convex function of our algorithm tuning factor $\alpha$, and iii) the optimum $\alpha$ 
value providing the minimum difference to the optimum decreases as the number of flows increases.

As future work we plan to extend the FFR algorithm proposed in this paper so that $\alpha$ does not need to be configured by the operator but is found dynamically in a self-organizing way, automatically adapting to variations in the number of service flows and changing channel conditions. Furthermore, the algorithm has to be extended to support different QoS classes.

\section{References}

1. Boudreau, G., Panicker, J., Guo, N., Chang, R., Wang, N., Vrzic, S.: Interference Coordination and Cancellation for $4 \mathrm{G}$ Networks. IEEE Communications Magazine 47(4), 74-81 (2009)

2. WiMAX Forum, Mobile WiMAX - Part I: A Technical Overview and Performance Evaluation (August 2006)

3. 3GPP, TS 36.201 Evolved Universal Terrestrial Radio Access (E-UTRA); Long Term Evolution (LTE) physical layer; General description, 3GPP, Tech. Rep. (March 2009)

4. IEEE $802.16 \mathrm{~m}$ D1, DRAFT Amendment to IEEE Standard for Local and metropolitan area networks, Part 16: Air Interface for Broadband Wireless Access Systems, Advanced Air Interface (July 2009)

5. ITU-R, REPORT ITU-R M.2134, Requirements related to technical performance for IMT-Advanced radio interface(s), ITU-R, Tech. Rep. (2008)

6. Rappaport, T.S.: Wireless Communications - Principles and Practice. PrenticeHall, Englewood Cliffs (2002)

7. Zander, J.: Generalized Reuse Parititioning in Cellular Mobile Radio. In: Proc. of 43rd IEEE VTC, May 1993, pp. 181-184 (1993)

8. Jia, H., Zhang, Z., Yu, G., Cheng, P., Li, S.: On the Performance of IEEE 802.16 OFDMA System Under Different Frequency Reuse and Subcarrier Permutation Patterns. In: Proc. of IEEE ICC '07, June 2007, pp. 5720-5725 (2007)

9. Godlewski, P., Maqbool, M., Klif, J.-M., Coupechoux, M.: Analytical Evaluation of Various Frequency Reuse Schemes in Cellular OFDMA Networks. In: Proc. of ACM ValueTools, October 2008. ICST, Athens (2008)

10. Zhou, Y., Zein, N.: Simulation Study of Fractional Frequency Reuse for Mobile WiMAX. In: Proc. of IEEE VTC Spring 2008, Singapore, May 2008, p. 2595 (2008)

11. Sarperi, L., Hunukumbure, M., Vadgama, S.: Simulation Study of Fractional Frequency Reuse in WiMAX Networks. Fujitsu Scientific and Technical Journal 44(3), 318-324 (2008)

12. IEEE 802.16 Rev 2, Part 16: Air Interface for Broadband Wireless Access Systems (May 2009)

13. Balachandran, K., Calin, D., Cheng, F.-C., Joshi, N., Kang, J.H., Kogiantis, A., Rausch, K., Rudrapatna, A., Seymour, J.P., Sun, J.: Design and Analysis of an IEEE 802.16e-Based OFDMA Communication System. Bell Labs Technical Journal 11(4), 53-73 (2007) 